\title{
Effet de l'association estradiol-17 $\beta$ et acétate de trenbolone sur la concentration plasmatique de certains métabolites et hormones chez le chevreau avant un repas
}

\author{
P Schmidely, P Bas, A Rouzeau \\ Station de Nutrition et Alimentation (INRA) de I'INA-PG, \\ 16, rue C-Bernard, 75231 Paris Cedex 05, France
}

\begin{abstract}
Summary - A combined implant of $17 \beta$-estradiol trenbolone acetate in young male goats decreased blood glucose and insulin $28 d$ after treatment, but had no effect on growth or thyroid hormones. No relation was found between hormonal concentrations and growth performance.
\end{abstract}

Introduction - Le mode d'action des anabolisants stéroïdiens est encore mal expliqué malgré leur effet bien connu sur la croissance chez les ovins, les bovins et aussi les caprins (Schmidely et al, 1987). Cet essai tente de déterminer les modifications de concentration plasmatique de certains métabolites et hormones, induites par l'implantation d'estradiol-17 $\beta$ combiné à l'acétate de trenbolone chez le chevreau mâle nourri au lait, et de les relier aux performances de croissance.

Matériel et Méthodes - Trente six chevreaux mâles sont répartis en 2 lots à 14 j d'âge : ils reçoivent en 2 repas/j un lait riche soit en lipides (lot $L$ ), ou en glucides (lot $G$ ) apportant respectivement par $\mathrm{kg}$ de matière sèche (MS): 4870 et 4750 kcal EM (énergie métabolisable) et 232 et $227 \mathrm{~g}$ de MAT (matières azotées totales). Après une période d'adaptation de $8 \mathrm{j}, 12$ animaux de chaque lot sont implantés avec $2,5 \mathrm{mg}$ d'estradiol-17 $\beta$ (E-17 $\beta$ ) et $17,5 \mathrm{mg}$ d'acétate de trenbolone (TBA) (lot IL, IG), les 6 chevreaux restant étant non traités (lot TL, TG). Les chevreaux subissent une prise de sang à jeûn, à l'âge de 7 semaines, le matin de leur abattage, afin de mesurer la concentration plasmatique en hormone de croissance (GH), insuline (INS), triïodo- et tétraïodothyronine $\left(T_{3}\right.$ et $T_{4}$ ) ainsi que les teneurs en acides gras non es- térifiés (AGNE), glucose (GLU) et urée (URE).

Résultats et Discussion - L'ingestion de $M S(g / j)$ de lait est réduite pendant les 28 j séparant traitement et abattage: 389 $(s=15), 355(s=35), 424(s=35), 387$ $(s=45)(P<0,05)$ pour les lots TL, IL, TG et IG respectivement, l'effet le plus important se produisant dans les 3 semaines après la pose de l'implant. Durant la période séparant implantation et traitement, les vitesses de croissance $(g / j)$ ne différent pas significativement entre les lots: $246(s=46), 251(s=62), 250(s=16)$ et $264(s=45)$. Dans nos conditions expérimentales, les anabolisants ou le régime ne modifient pas la concentration plasmatique en GH (tableau 1), ce qui est comparable aux résultats de Galbraith et Watson (1978) chez le boeuf. Ceci peut être attribué au délai entre la pose de l'implant et la prise de sang mais aussi aux variations individuelles intra- et inter-jour de la concentration en $\mathrm{GH}$. La concentration en hormones thyroïdiennes n'est modifiée ni par le régime, ni par les anabolisants contrairement aux observations de Toullec et Manis (1986) chez le veau. Les che- 
Tableau 1 . Effet des anabolisants et du régime sur les concentrations plasmatiques de certains métabolites et hormones. ${ }^{1}$

\begin{tabular}{|c|c|c|c|c|c|c|c|c|}
\hline \multirow[t]{2}{*}{ Traitement } & \multicolumn{4}{|c|}{ Témoin } & \multicolumn{4}{|c|}{ Implant } \\
\hline & \multicolumn{2}{|c|}{ lipides } & \multicolumn{2}{|c|}{ glucides } & \multicolumn{2}{|c|}{ lipides } & \multicolumn{2}{|c|}{ glucides } \\
\hline $\begin{array}{l}\mathrm{GH}(\mathrm{ng} / \mathrm{ml}) \\
\text { Insuline }(\mu \mathrm{U} / \mathrm{ml}) \\
\mathrm{T}_{3} \text { (ng/ml) } \\
\mathrm{T}_{4}(\mathrm{ng} / \mathrm{ml}) \\
\text { Glucose }(\mathrm{g} /) \\
\text { Urée }(\mathrm{g} / \mathrm{l}) \\
\text { AGNE }(\mu \text { eq/) } \\
\text { Engraissement }{ }^{2}\end{array}$ & $\begin{array}{c}8,6 \\
71 \\
2,3 \\
56 \\
1,06 \\
0,27 \\
280 \\
3,4\end{array}$ & $\begin{array}{c}(5,6) \\
(39)^{\mathrm{a}} \\
(0,3) \\
(6) \\
(0,06)^{\mathrm{a}} \\
(0,07) \\
(110)^{\mathrm{a}} \\
(0,5)^{\mathrm{a}}\end{array}$ & $\begin{array}{c}4,9 \\
143 \\
2,1 \\
48 \\
1,06 \\
0,24 \\
190 \\
3,1\end{array}$ & $\begin{array}{c}(3,8) \\
(50)^{\mathrm{b}} \\
(0,4) \\
(10) \\
(0,13)^{\mathrm{a}} \\
(0,01) \\
(190)^{\mathrm{b}} \\
(0,4)^{\mathrm{a}}\end{array}$ & $\begin{array}{c}5,7 \\
30 \\
2,4 \\
58 \\
0,98 \\
0,24 \\
207 \\
2,4\end{array}$ & $\begin{array}{c}(3,4) \\
(21)^{\mathrm{c}} \\
(0,6) \\
(10) \\
(0,09)^{\mathrm{b}} \\
(0,05) \\
(69)^{\mathrm{b}} \\
(0,5)^{\mathrm{b}}\end{array}$ & $\begin{array}{c}7,6 \\
42 \\
2,3 \\
55 \\
1,00 \\
0,23 \\
180 \\
2,6\end{array}$ & $\begin{array}{c}(7,6) \\
(25)^{\mathrm{d}} \\
(0,3) \\
(10) \\
(0,10)^{\mathrm{a}} \\
(0,04) \\
(84)^{\mathrm{b}} \\
(0,6)^{\mathrm{b}}\end{array}$ \\
\hline
\end{tabular}

\footnotetext{
1 Valeurs exprimées par moyenne (écart type). Les valeurs non affectées de méme lettres diffèrent significativement $(P<0,05) .{ }^{2}$ Noté de 1 à $5: 1$ = maigre à $5=$ gras.
}

vreaux traités par les anabolisants présentent une forte réduction de leur insulinémie, par ailleurs plus importante dans le lot $\mathrm{G}$. Parallèlement, comme la relation entre GLU et INS est significative ( $r$ (GLU, INS) $=+0,55, P<0,01$ ), on observe une réduction par les anabolisants de la glycémie de même amplitude quel que soit le régime. Ces 2 paramètres sont probablement l'une des raisons de la diminution de l'état d'engraissement observée chez les animaux traités. L'urémie sanguine n'est modifiée ni par le régime ni par les anabolisants, ce qui correspond à nos résultats précédents (Schmidely et al, 1988) où nous avons montré que la réduction de l'urémie liée à une rétention azotée améliorée par $\mathrm{E}-17 \boldsymbol{\beta}+\mathrm{TBA}$ ne durait que 3 semaines environ après la pose de l'implant. Aucune relation significative n'a pu être mise en évidence entre ces paramètres sanguins instantanés et les performances de croissance mesurées sur la période expérimentale ou durant les $7 \mathrm{j}$ précédant l'abattage.

En conclusion, chez le chevreau nourri au lait, l'implantation d'estradiol-17 $\beta$ combiné à TBA induit peu d'effets sur la vitesse de croissance, mais réduit l'ingestion et l'état d'engraissement. Aucune de ces modifications n'a pu être reliée à des variations de paramètres hormonaux ou métaboliques du plasma, excepté la réduction de l'état d'engraissement qui pourrait être due à la diminution de l'insuline.

Galbraith H, Watson H (1978) Vet Rec 103, 28-30

Schmidely P, Morand-Fehr P, Bas P, Hervieu J (1987) Ann Zootech 36, 343

Schmidely P, Bas P, Rouzeau A (1988) Séminaire FAO Goat Feeding and Nutrition, Potenza, Italie, Oct 1988

Toullec R, Mannis Y (1986) Bull Tech CRZV Theix $65,25-30$ 